\title{
EXPLORING CONFIDENTIAL DEDUPLICATION OF DATA USING BIG DATA IN CLOUD COMPUTING STORAGE
}

\author{
Thangarasan $\mathrm{T}$, \\ Department of CSE \\ K.S.R College of \\ Engineering \\ (Autonomous), \\ Tiruchengode, \\ Namakkal Dt, \\ Tamilnadu, India.
}

\author{
Jaikumar B, \\ Department of CSE \\ Paavai College of \\ Engineering, \\ Rasipuram, \\ Namakkal Dt, \\ Tamilnadu, India.
}

\author{
Keerthana R, \\ Department of CSE \\ Sengunthar \\ Engineering College \\ (Autonomous), \\ Tiruchengode, \\ Namakkal Dt, \\ Tamilnadu, India.
}

\author{
Prakash P, \\ Department of CSE \\ Paavai College of \\ Engineering, \\ Rasipuram, \\ Namakkal Dt, \\ Tamilnadu, India.
}

\begin{abstract}
To decrease the selecting time and reaction time between Token deals and reaction, File move or download mentioning and results. It diminishes the extent of extra room in flowed limit. To ensure about the assurance of information differential confirmed copy check is utilized. It presents this supported copy check in half and half cloud structure. The mutt cloud arrangement proposes about both the open cloud and the private cloud. So as to give progressively conspicuous security, the private cloud is equipped with staggered check. Kinds of progress in scattered figuring are inciting a promising future for Collaborative Cloud Computing (CCC). To decrease the getting ready time and reaction time between Token deals and reaction, File move or download mentioning and results. Where comprehensive dispersed dissipated cloud assets having a spot with various affiliations or people (i.e., segments) are aggregately utilized in a strong strategy to give organizations. The records are dealt with in the cloud. That is each customer selects an information key to encode the information that he plans to store in the cloud. It depicts a computationally unassuming procedure for making all log portions made. Going before the logging machine's trade off unfathomable for the assailant to examine and besides hard to elusively adjust or wreck. Each Client computes an information key to encode the information that he would like to store in the cloud.
\end{abstract}

Keywords - Collaborative Cloud Computing (CCC), AES, MD5 and SHA Algorithm.

\section{INTRODUCTION}

To reduce the figuring time and reaction time between token mentioning and reaction, File move or download deals and results. It decreases the extent of extra room in passed on limit. To ensure about the riddle of information differential supported copy check is utilized. It present this embraced copy check in cross breed cloud building. The half and half cloud building proposes about both the open cloud and the private cloud. So as to give increasingly significant security, the private cloud is equipped with shocked check. Kinds of progress in appropriated preparing are instigating a promising future for Collaborative Cloud Computing (CCC). To diminish the enlisting time and reaction time between Token deals and reaction, File move or download mentioning and results. Where completely dissipated passed on cloud assets having a spot with various affiliations or people (i.e., parts) are everything seen as utilized in a lovely way to offer sorts of help. The records are dealt with in the cloud. That is each customer computes an information key to encode the information that he expects to store in the cloud. It depicts a computationally subtle method for making all $\log$ sections made. Before the logging machine's trade off staggering for the aggressor to analyze and in addition hard to indistinguishably change or pound. That is each customer enrolls an information key to scramble the information that he would like to store in the cloud. Information De-duplication with focus is one of colossal information revealing structures for taking copy duplicates of emphasizing information. It looked at the extent of extra room and additional trade speed. To ensure the puzzle of touchy information while supporting De-duplication with focus point, the concurrent encryption method has been proposed to scramble the information before redistributing. We propose another instigated duplication structure supporting embraced copy check and contrast the breaking point framework and record substance. The crossbreed cloud arrangement proposes about both the open cloud and the private cloud. At the present time, information duplicates of various clients will incite grouped figure works, making De-duplication with focus tremendous. To give 
increasingly critical security, the private cloud is equipped with stunned check.

\section{PROBLEM STATEMENT}

The Main purpose of deduplication to give security on social destinations keeping up a key good ways from different copies of same data with the objective that any issues arise the copy of the data can be ousted.

\section{EXISTING SYSTEM}

The mixed encryption technique has been proposed to scramble the information before re-appropriating. To even more likely assurance information security, this framework makes the head undertaking officially address the issue of supported information De-duplication. Undeniable filename reliant on the differential favourable circumstances of clients are likewise considered in copy check report name trademark the information itself. It moreover shows a few new Deduplication headways supporting certified copy. Information managing in the cloud experiences an eccentric and dynamic diverse levelled to association chain. This doesn't exist in conventional conditions. Standard web structure Uses web associations for mentioning and reactions.

\section{* Disadvantages}

$\checkmark$ This conventional joined encryption will be flimsy for clear report.

$\checkmark$ There might be a corresponding record name rehashed it may fight.

\section{PROPOSED SYSTEM}

An Another moved duplication structure supporting embraced copy check and intricacy the breaking point framework and report content. Right now the structure, the private keys for benefits won't be given to clients plainly which will be kept and coordinated by the private cloud server. The information will be blended utilizing AES estimation. Right now, clients can't move a similar hash respect information since it analyzes the entire information base which recommends that it can forestall the duplication methodology with same substance. To get a report respect, the client needs to send a mentioning to the private cloud server. To play out the copy check for some record by the Comparison the breaking point framework, the client needs to get the document content from the cloud server. The embraced copy check for this report substance can be performed by the MD5 and SHA figuring in the server putting away before moving this record. Considering the aftereffects of copy check the client either moves this record.

\section{* Advantages of Proposed System}

$\checkmark$ Encryption of information is finished utilizing AES estimation

$\checkmark$ The basic thought of our system is that the novel encryption key age estimation.

$\checkmark$ Ensure the information security by remembering differential points of interest of clients for the copy check.

$\checkmark$ Get effective thing set outcome dependent on the Deduplication.

$\checkmark \quad$ MD5 and sha1 calculation is utilized to perceive copy duplicates.

\section{RELATED WORK}

\section{Client Enrollment}

The customer should move toward agree to executive for customer selection. At the point when director gives assent then OTTP will be send through User Email. Using that OTTP the customer needs to enroll.

\section{Document Upload}

For storing a data report, the customer can move many record, while the archive send to the server will be encoded using AES Algorithm for Security purposes. The developer can't hack the archive while moving so it is encoded using AES Algorithm with the objective that no issues of hacking occurs.

\section{Key Comparison}

In the wake of moving record, for each archive key will be made using MD5 and SHA Algorithm. Keys will be taken care of in hash table for connection purposes. With the Key of the report is stood out from other record keys for keep up single copy of data. With the ultimate objective that any issue rises, single copy can be viably removed.

\section{Root Priority}

The User who at first exchanges a record will be the essential root center point, by then the ensuing who moves a comparable report will the consequent center point, third who moves a comparative archive will be the third center so on. Expect the important customer who moves the record deletes the copy then the resulting who moves a comparable archive will be base of the center. 
International Journal of Engineering Applied Sciences and Technology, 2020

Vol. 5, Issue 8, ISSN No. 2455-2143, Pages 111-114

Published Online December 2020 in IJEAST (http://www.ijeast.com)

\section{SYSTEM ARCHITECTURE}

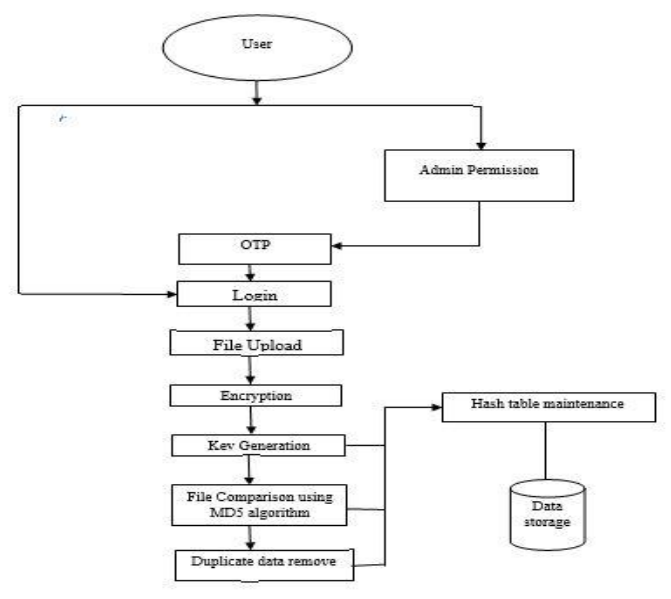

VII. HASH VALUE GENERATOR ALGORITHMS

A Hash Value (likewise called as Hashes or Checksum) is a string estimation (of explicit length), which is the consequence of figuring of a Hashing Algorithm. Hash Values have various employments. One of the principle employments of Hash Values is to decide the Integrity of any Data (which can be a record, envelope, email, connections, downloads and so forth). In the event that you need to perceive how a Hash Value resemble, visit next exercise How Hash Values can be utilized to decide Integrity of Data

The most superb character of Hash Values is that they are profoundly novel. No two information can hypothetically have same Hash Value.

There is a condition called as Collision in Hashing. Impact is a circumstance when two distinct Data have a similar Hash Value. Best hashing calculation is the one which can't cause Hash Value Collision.

Significant Hashing Algorithms are recorded beneath.

MD5 (Message Digest, characterized by RFC 1321) - MD5 Hashing Algorithm was designed by RSA Labs (Ronald Rivest) in 1991. MD5 was developed to supplant its past rendition, MD4. At the point when Data is taken care of to MD5 Hashing Algorithm, it creates a 128-piece Hash Value String as a 32 digit hexadecimal number. Hash Value Collisions are accounted for MD5 Hashing Algorithm.

\section{EXPERIMENT \& RESULTS}

The following figures shows the results of image has the hash value and it is denoted only on the root. If the image is deleted by the root then automatically the second uploaded person get the hash value of the image Fig (a) represents Login process. Fig (b),(c) shows File uploading. Fig (d) Shows the hash values and root priority of the image which is uploaded by distinct users. Fig (e) shows the Root Priority (i.e) the person who uploaded initially was deleted so the hash value moved to the person who uploaded next.

User Login

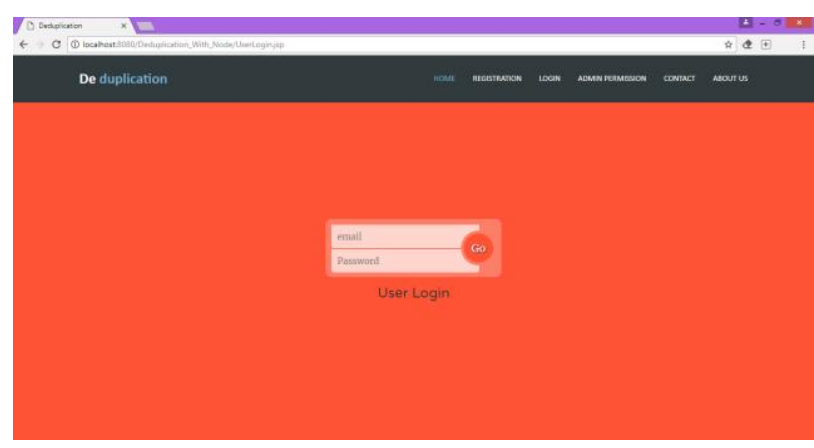

File Upload

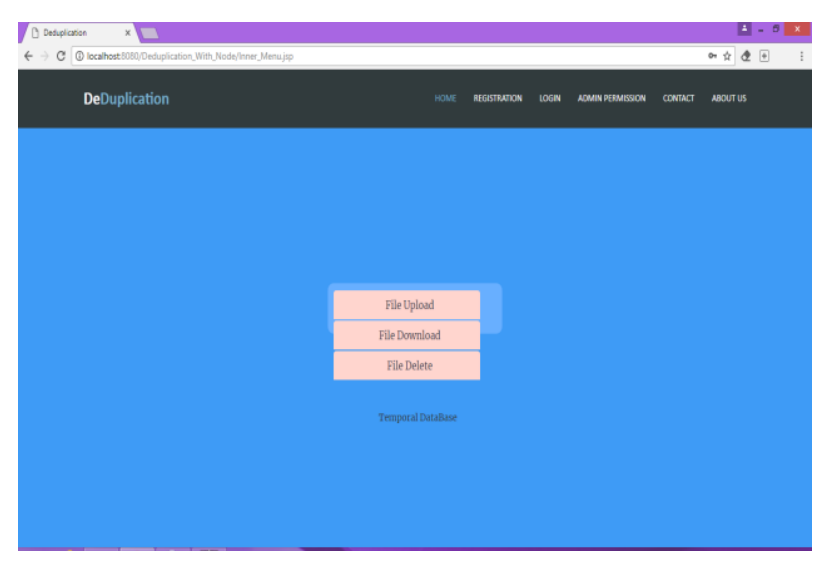

File Uploading

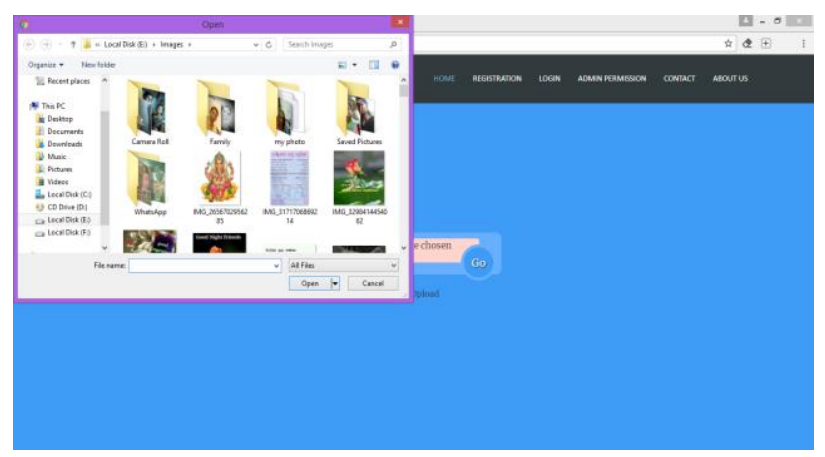




\section{Hash Values(Before Delete)}

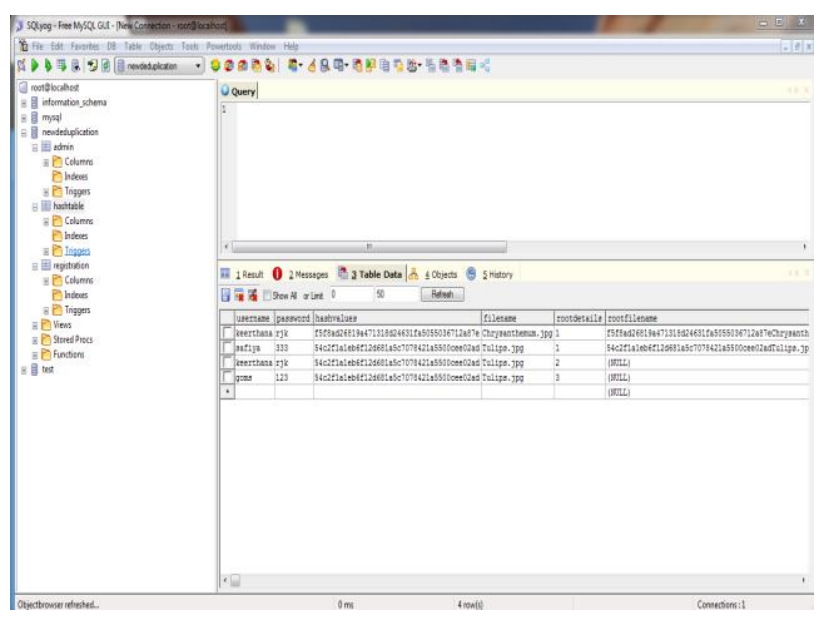

Hash Values(After Delete)

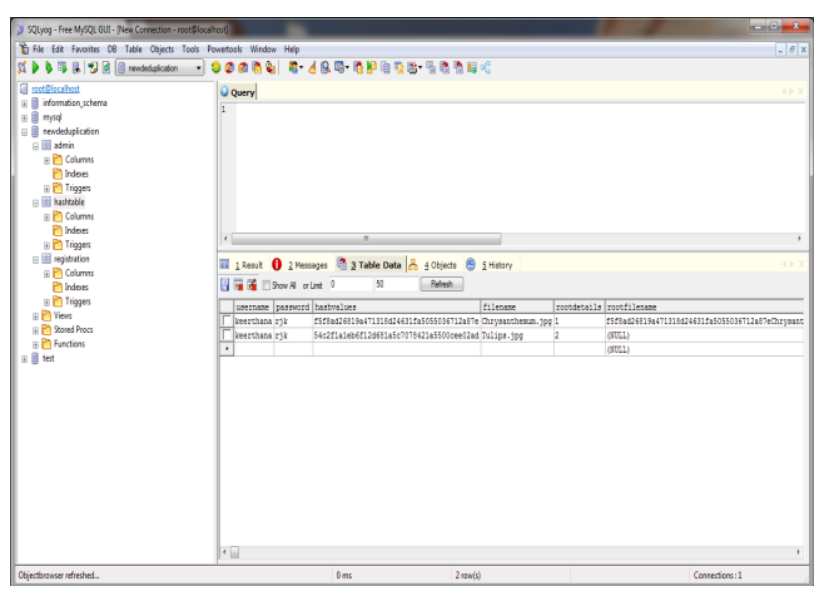

\section{CONCLUSION}

The as of late proposed system is done structure to securely refitting $\log$ records to a cloud provider. At this moment, out the challenges for a sheltered cloud-based log the administrator's organization. The aggressors use underneath three phases to hack. In any case, the attacker can obstruct any message sent over the Internet. Second, the attacker can fuse, copy, and replay messages in his possession and the assailant can be a genuine individual from the framework or can endeavor to impersonate authentic hosts. It executes how to store secure log record in cloud and that report we can change read, make, delete, move and download. It can execute AES computation that uses for $\log$ screen and $\log$ generator. One of these extraordinary troubles is the issue of log assurance that rises when we re-appropriated $\log$ the officials to the cloud. Log information right now not be coolly linkable or perceivable to their sources during limit, recuperation and crossing out. It gave obscure exchange, recoup and delete shows on log records in the cloud using the Tor orchestrate. The shows that it made consequently have potential for use in a wide scope of zones incorporating strange circulate purchase in.

\section{REFERENCES}

[1] Gagangeet Singh Aujla, Rajat Chaudhary, Neeraj Kumar, Ashok Kumar Das, and Joel J. P. C. Rodrigues, (2018). 'SecSVA: Secure Storage, Verification, and Auditing of Big Data in the Cloud Environment'.

[2] Qian Wang, Student Member, IEEE, Cong Wang, Student Member, IEEE, Kui Ren, Member, IEEE, Wenjing Lou, Senior Member, IEEE, and Jin Li, (2011). 'Enabling Public Auditability and Data Dynamics for Storage Security in Cloud Computing'.

[3] Jingwei Li, Jin Li, Dongqing Xie and Zhang Cai, (2016). 'Auditing and Deduplicating Data in Cloud'.

[4] Huiying Hou, Jia Yu, Rong Hao, (2019). 'Cloud storage auditing with deduplication supporting different security levels according to data popularity'.

[5] Hui Tian, Member, IEEE, Yuxiang Chen, Chin-Chen Chang, Fellow, IEEE,Hong Jiang, Fellow, IEEE, Yongfeng Huang, Senior Member, IEEE, Yonghong Chen, Member, IEEE, Jin Liu, Member, IEEE , (2016). 'Dynamic-Hash-Table Based Public Auditing for Secure Cloud Storage'.

[6] Jing Hana, Yanping Li, Weifeng Chenb, (2018). 'A Lightweight And privacy-preserving public cloud auditing scheme without bilinear pairings in smart cities'.

[7] Shai Halevi IBM T. J. Watson, (2011). 'Proofs of Ownership in Remote Storage Systems'.

[8] Giuseppe Ateniese Randal Burn Reza Curtmola Joseph Herring Lea Kissner Zachary Peterson Dawn Song, (2015). 'Provable Data Possession at Untrusted Stores'.

[9] Jiawei Yuan (2013). 'Secure and Constant Cost Public Cloud Storage Auditing with Deduplication'.

[10] Cong Wang, Qian Wang, and Kui Ren (2017). 'PrivacyPreserving Public Auditing for Data Storage Security in Cloud Computing'.

[11] P. Lu et al., (2015). 'Highly Efficient Data Migration and Backup For Big Data Applications In Elastic Optical Inter-Data-Center Networks', IEEE Network, vol. 29, no. 5, pp. 36-42.

[12] W. Stallings, (2006). 'Cryptography and Network Security Principles and Practices', I. Pearson Education, Pearson Prentice Hall. 